\title{
Schwannoma of the colon: A case report and review of the literature
}

\author{
WEI-BING WANG, WEN-BIN CHEN, JIAN-JIANG LIN, JIA-HE XU, JIN-HAI WANG and QIN-SONG SHENG \\ Department of Colorectal and Anal Surgery, The First Affiliated Hospital, Zhejiang University School of Medicine, \\ Hangzhou, Zhejiang 310003, P.R. China
}

Received January 18, 2015; Accepted February 12, 2016

DOI: $10.3892 / \mathrm{ol} .2016 .4271$

\begin{abstract}
Colonic schwannomas are rare gastrointestinal mesenchymal tumors, and only a limited number of cases has been reported. The occurrence of these tumors is less common in the large intestine than in the stomach. The present study reports a case of colonic schwannoma in a 62-year-old female patient with no specific symptoms. The patient was diagnosed with a mass in the ascending colon by colonoscopy and abdominal computed tomography scanning. A right hemicolectomy was performed. The postoperative pathological diagnosis was ascending schwannoma. This case is noteworthy as colonic schwannomas are rare and are typically treated as colon cancer. No recurrence of the lesion was observed after 24 months of follow-up.
\end{abstract}

\section{Introduction}

Schwannomas are tumors originating from Schwann cells. This type of tumor may be found throughout the body along the peripheral nerves; however schwannomas of the colon and rectum are extremely rare (1). This rare tumor accounts for $2-6 \%$ of all mesenchymal tumors (2). The incidence rates of schwannoma are identical for men and women, and the age of such patients is between 60 and 65 years (3). Due to the small number of such cases, the characteristics of this tumor are not fully established (4). Immunohistochemistry of the tumor cells remains the most important diagnostic method. When the tumor is located in the colon or in the rectum, radical excision with wide margins is mandatory, due to its tendency to recur locally or become malignant if left untreated. The surgical approach depends on the size, location and histopathological pattern of the tumor (4). The use of radiotherapy or adjuvant

Correspondence to: Mr. Qin-Song Sheng, Department of Colorectal and Anal Surgery, The First Affiliated Hospital, Zhejiang University School of Medicine, 79 Qingchun Road, Hangzhou, Zhejiang 310003, P.R. China

E-mail: shengqinsong@163.com

Key words: schwannoma, colon, gastrointestinal tumor chemotherapy has conflicting results and is not recommended for routine use. The present study reports a rare case of a schwannoma present in the ascending colon that was detected by colonoscopy and abdominal computed tomography (CT) scanning, and required surgical resection.

\section{Case report}

A 62-year-old female patient was admitted to The First Affiliated Hospital of Zhejiang University School of Medicine (Hangzhou, China), presenting with abdominal pain and a history of intermittent, dark-red bloody stools for 1 month. The patient's medical history included uterine fibroids and hypertension. The patient had no other specific medical conditions, including neurofibromatosis. There was no family history of inflammatory bowel disease or cancer, and she had had no prior abdominal surgeries. A physical examination revealed mild tenderness in the right lower quadrant. The laboratory test results were normal. A colonoscopy (Olympus Corporation, Toyko, Japan) revealed a pedunculated mass in the proximal ascending colon measuring $\sim 4 \times 4 \mathrm{~cm}$ (Fig. 1). No lesions were found in the other colon segments, including the cecum. An abdominal CT scan (Aquilion 16; Toshiba, Tokyo, Japan) revealed a round, homogeneous, low-attenuation mass in the proximal ascending colon, without adjacent wall thickening (Fig. 2). No enlarged pericolic lymph nodes were observed.

The patient underwent a right hemicolectomy without a preoperative endoscopic biopsy. The procedure involved removal of the bowel from 4-6 cm proximal to the ileocecal valve to the portion of the transverse colon supplied by the right branch of the middle colic artery. An anastomosis was fashioned between the terminal ileum and the transverse colon. No infiltration or distant dissemination was identified. The resected specimens were fixed with $10 \%$ formalin fixative and 95\% ethanol fixative, dehydrated, embedded in wax, sectioned and stained with hematoxylin and eosin (Leica Microsystems, Inc., Buffalo Grove, IL, USA). Microscopic analysis revealed that the tumor was composed of proliferating spindle cells arranged in fascicular or vague palisading patterns, in a loose edematous stroma with inflammatory cell infiltration (Fig. 3; hematoxylin and eosin stain). Immunohistochemical analysis revealed that the tumor was positive for S-100; however, no reactivity for cluster of differentiation (CD) 117, CD34, desmin, smooth muscle actin or discovered on gastrointestinal stromal 


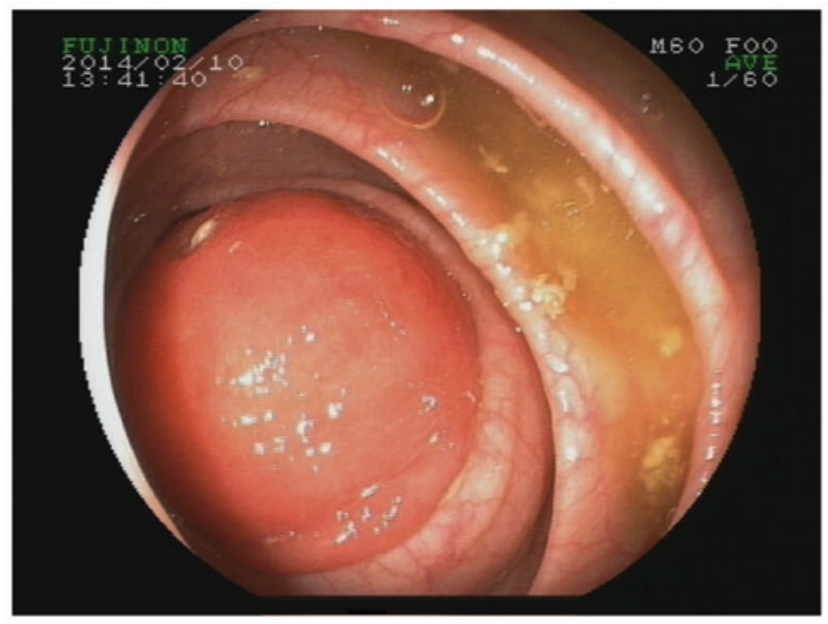

Figure 1. Colonoscopy image showing a pedunculated mass measuring $\sim 4 \mathrm{x} 4 \mathrm{~cm}$ in the proximal ascending colon.

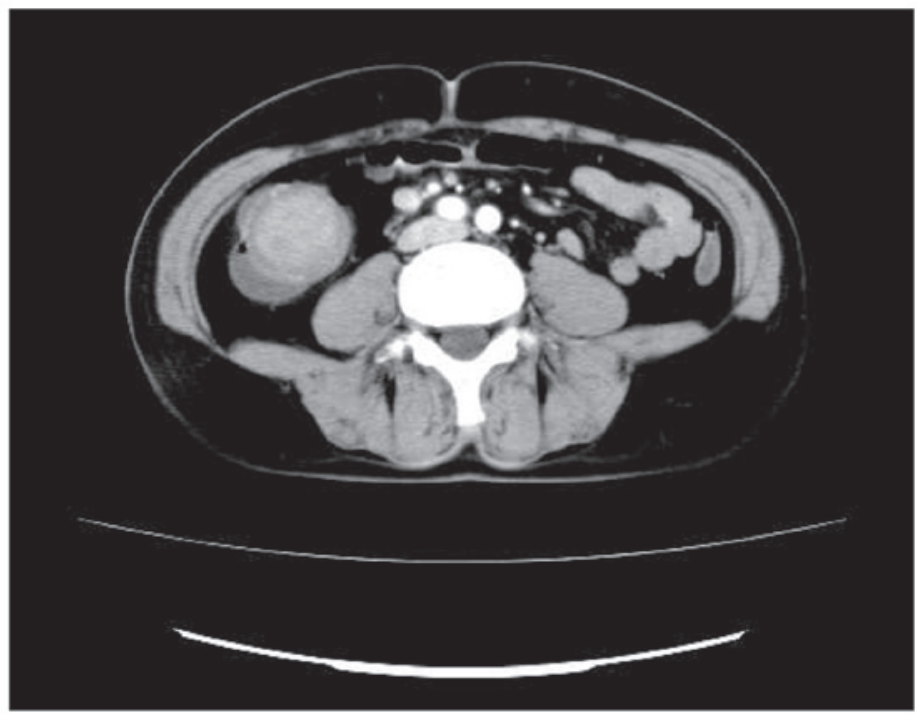

Figure 2. Computed tomography scan revealing a round, homogeneous, low-attenuation mass in the proximal ascending colon without adjacent wall thickening.
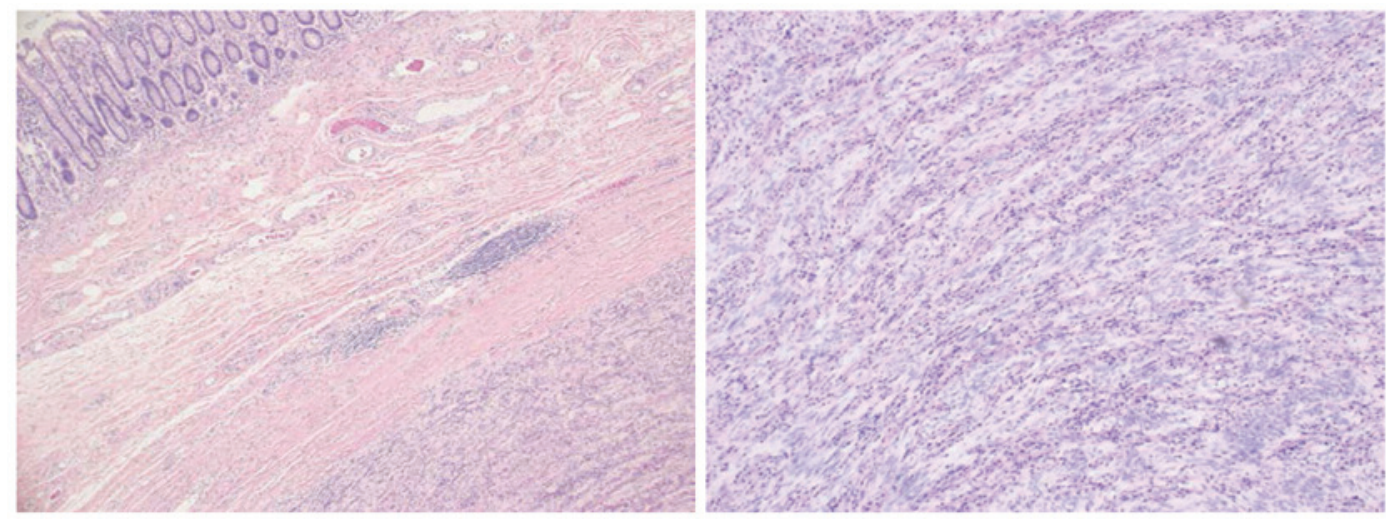

Figure 3. Immunohistochemical analysis showed that the tumor was composed of a proliferation of spindle cells arranged in fascicular or vague palisading patterns in a loose edematous stroma with inflammatory cell infiltration. Hematoxylin and eosin staining; magnification, left panel x200 and right panel x50.

tumor-1 was detected. Ki-67 labeling was observed in $<3 \%$ of the tumor cells. The definitive diagnosis was schwannoma of the colon. No recurrence was observed during the 24-month follow-up period and no additional treatment was administered.

\section{Discussion}

Verocay first described schwannomas in 1910 (4). Despite the increasing number of mesenchymal tumor reports with the 
advent of modern immunohistochemical staining techniques, primary schwannomas of the colon and rectum that are not associated with systemic neurofibromatosis (von Recklinghausen disease) are extremely rare $(5,6)$. Due to the small number of such cases, the incidence rates and characteristics of schwannomas have not been fully determined. Schwannomas are known to be benign neoplasms of ectodermal origin, which are characterized by slow growth and the capability for malignant degeneration if not removed (5-7). This type of tumor typically manifests as a polyp that may ulcerate the mucosa $(8,9)$, leading to nonspecific symptoms, including abdominal pain with rectal bleeding, defecation disorders and colonic obstruction or invagination, as in the present case $(10,11)$. Imaging findings are nonspecific; CT scans show well-defined, homogeneous mural masses, and can help to distinguish schwannomas from gastrointestinal stromal tumors (GISTs), which are heterogeneous masses (12). On most occasions, diagnosis is not established based on a biopsy but on a surgical specimen (13).

Macroscopically, schwannomas are well-circumscribed, yellowish-white lesions (4). Immunohistochemical examination of the tumor cells is considered the optimal diagnostic tool for this type of tumor (14). Schwannomas usually exhibit positive reactivity for S-100, vimentin and glial fibrillary acidic protein, and no reactivity for CD117, CD34, actin or cytokeratins, which appear more typically in GISTs, gastrointestinal autonomic tumors or muscle tumors $(15,16)$. Following diagnosis, treatment options include polypectomy or segmental colectomy with free margins due to the low risk of malignancy $(17,18)$. The benign nature of the tumor is responsible for the good prognosis of patients with schwannoma; recurrence and metastasis are considered rare events. In conclusion, colonic schwannoma is a rare tumor with a benign behavior and patients with this type of tumor have a favorable prognosis.

\section{Acknowledgements}

This study was supported by the Natural Science Foundation of Zhejiang Province (grant no. LY13H030004).

\section{References}

1. Zippi M, Pica R, Scialpi R, Cassieri C, Avallone EV and Occhigrossi G: Schwannoma of the rectum: A case report and literature review. World J Clin Cases 1: 49-51, 2010.

2. Verdú-Fernández MÁ, Guillén-Paredes MP, García-García ML, García-Marín JA, Pellicer-Franco E and Aguayo-Albasini JL: Schwannoma in descending colon: Presentation of a neoplasm in a rare location. Rev Esp Enferm Dig 105: 502-503, 2013.
3. Miettinen M, Sarlomo-Rikala M and Lasota J: Gastrointestinal stromal tumours. Ann Chir Gynaecol 87: 278-281, 1998.

4. Baek SJ, Hwangbo W, Kim J and Kim IS: A case of benign schwannoma of the ascending colon treated with laparoscopic-assisted wedge resection. Int Surg 98: 315-318, 2013.

5. Daimaru Y, Kido H, Hashimoto $H$ and Enjoji M: Benign schwannoma of the gastrointestinal tract: A clinicopathologic and immunohistochemical study. Hum Pathol 19: 257-264, 1988.

6. Nonose R, Lahan AY, Santos Valenciano J and Martinez CA Schwannoma of the colon. Case Rep Gastroenterol 3: 293-299, 2009.

7. Lauwers GY, Erlandson RA, Casper ES, Brennan MF and Woodruff JM: Gastrointestinal autonomic nerve tumors: A clinicopathological, immunohistochemical and ultrastructural study of 12 cases. Am J Surg Pathol 17: 887-897, 1993.

8. Kwon MS, Lee SS and Ahn GH: Schwannomas of the gastrointestinal tract: Clinicopathological features of 12 cases including a case of esophageal tumor compared with those of gastrointestinal stromal tumors and leiomyomas of the gastrointestinal tract. Pathol Res Pract 198: 605-613, 2002.

9. Tanaka T, Ishihara Y, Takabayashi N, Kobayashi R, Hiramatsu T and Kuriki K: Gastrointestinal: Asymptomatic colonic schwannoma in an elderly woman; a rare case. J Gastroenterol Hepatol 26: 1339, 2011.

10. Martínez Crespo JJ, Vicente JJ, García Pérez B, Pérez Guillermo M and González Costea R: Atipic manifestation of an infrequent lesion. Video- and ecoendoscopy in a gastric schwannoma. Rev Esp Enferm Dig 97: 844-845, 2005.

11. Kim HJ, Kim CH, Lim SW, Huh JW, Kim YJ and Kim HR: Schwannoma of ascending colon treated by laparoscopic right hemicolectomy. World J Surg Oncol 10: 81, 2012.

12. Levy AD, Quiles AM, Miettinen M and Sobin LH: Gastrointestinal schwannomas: CT features with clinicopathologic correlation. AJR Am J Roentgenol 184: 797-802, 2005.

13. Yoon W, Paulson K, Mazzara P, Nagori S, Barawi M and Berri R: Gastric schwannoma: A rare but important differential diagnosis of a gastric submucosal mass. Case Rep Surg 2012: 280982, 2012.

14. Arai T, Sugimura H, Suzuki M, Iwase T, Sakuramachi S, Kimura T, Harada Y and Kino I: Benign schwannoma of the esophagus: Report of two cases with immunohistochemical and ultrastructural studies. Pathol Int 44: 460-465, 1994.

15. Torres Gómez FJ, Fernández Machín P, del Álamo Juzgado C, Martínez A, Martínez Moyano1 A, Moreno Corral S: Schwannoma quístico de colon. Presentación de un caso. Rev Esp Patol 42: 143-146, 2009.

16. Hou YY, Tan YS, Xu JF, Wang XN, Lu SH, Ji Y, Wang J and Zhu XZ: Schwannoma of the gastrointestinal tract: A clinicopathological, immunohistochemical and ultrastructural study of 33 cases. Histopathology 48: 536-545, 2006.

17. Park KJ, Kim KH, Roh YH, Kim SH, Lee JH, Rha SH and Choi H: Isolated primary schwannoma arising on the colon: Report of two cases and review of the literature. J Korean Surg Soc 80: 367-372, 2011.

18. Watanabe A, Ojima H, Suzuki S, Mochida Y, Hirayama I, Hosouchi Y, Nishida Y, Kashiwabara K, Ohno T, Mochiki E and Kuwano H: An individual with gastric schwannoma with pathologically malignant potential surviving two years after laparoscopy-assisted partial gastrectomy. Case Rep Gastroenterol 5: 502-507, 2011. 